\title{
Critical Study on the Angular-momentum-transfer Method and Vorticity-transfer Method in the Investigation of Zonal Circulation of the Atmosphere
}

\author{
H. Arakawa \\ Muteorological Reseurch Institute, Suginami-kn, Tokyo. \\ (Manuscript received 3 March 1943)
}

\begin{abstract}
A comparative and critical study of the angular-momentum-transfer and vorticitytransfer methods in the investigation of zonal circulation of the atmosphere is given. The essential difference between the two methods is that the fluctuating variations of atmospheric pressure in the west-east direction, which certainly exist in a turbulent field of flow, are ignored in the former method, while in the latter the variations are. taken into account. According to the former method, the displaced particle moving. towards the pole gains the west component of wind speed and the displaced particlemoving towards the equator loses the west component of wind speed. While, according to the latter method, the displaced particle moving north or southward usually gains. the west component of wind speed. Very high velocities will result when a mass is moved horizontally from a given latitude towards the pole or equator under conservation of its angular momentum, while quite reasonable velocities will be given when a mass is moved borizontally under conservation of its absolute vorticity. Such an essential difference can be readily seen from Tables $1 \cdot a$ and $1 \cdot b$.
\end{abstract}

\section{$\S$ 1. Introduction}

The investigations hitherto have resulted in two different methods of investigating zonal circulation of the atmosphere. and in two sets of formulae for zonal current, stability;" etc. We shall refer to these meihods as the angular-momentum-transfer method and the vorticity-transfer method.

The essential difference between the angular-momentum-transfer method and the vorticity-transfer method is the following point: The assumption that angular momentum is a transferable property necessarily involves the assumption that the fluctuating variations in pressure, which certainly exist in a turbulent field of flow, are ineflective so far as the mean transport of angular momentum is concerned. On the other hand, if the turbulent motion is two-dimensional in the horizontal, the vertical component of absolute vorticity is conserved and transferable.

The difficulty in using the vorticity-transfer method in practice is due to the circumstance that the expressions for the excess wind speed of the displaced particle are rather complex. The aim of this paper is to make a comparative and critical study of the two theories of zonal circulation.

\section{\$ 2. Some theoretical relations of the angular momentum and the vorticity.}

The equations of horizontal motion in an atmosphere without friction are 


$$
\begin{gathered}
\frac{d v_{\lambda}}{d t}-\frac{\tan \phi}{r} v_{\phi} v_{\lambda}-2 \omega \sin \phi \cdot v_{\phi}=-\frac{1}{\rho \cdot r \cos \phi} \frac{\partial p}{\partial \lambda}, \\
\frac{d v_{\phi}}{d t}+\frac{\tan \phi}{r} v_{\lambda}{ }^{3}+2 \omega \sin \phi \cdot v_{\lambda}=-\frac{1}{\rho \cdot r} \frac{\partial p}{\partial \phi},
\end{gathered}
$$

where $r, \phi, \lambda$ are spherical polar coordinates, denoting respectively the radius, the latitude and the longitude; $v_{r}, v_{\phi}, v \lambda$ are the component velocities; $p$ is the pressure, $\rho$ the density, $t$ the time, $\omega$ the angular velocity of the earth's rotation, and $d / d t=\partial / \partial t+v_{\phi}(\partial / r \partial \phi)$ $+v_{\lambda}(\partial / r \cos \phi \partial \lambda)$.

The equation of continuity is

$$
\frac{d \rho}{d t}+\rho\left(\frac{\partial v_{\phi}}{r \partial \phi}-\frac{\tan \phi}{r} v_{\phi}+\frac{1}{r \cos \phi} \frac{\partial v_{\lambda}}{\partial \lambda}\right)=0
$$

For a steady, zonal current, we have from Eq. (2),

$$
2 \omega \sin \phi \cdot v_{\lambda}+\tan \phi \cdot v_{\lambda}^{2} / r=-\frac{1}{\rho} \frac{1}{r} \frac{\partial p}{\partial \phi} .
$$

The gradient wind $v_{\lambda}$ can be calculated from Eq. (3). This gradient wind equation is a quadratic and has two solutions. When the isobars are straight, the quadratic degenerates. into a simple equation whose solution is equal to the geostrophic wind. The solution of the quadratic which has a real physical meaning must therefore give the geostrophic wind, when the radius $r$ is infinitely increased. Thus we find

$$
v_{\lambda}=-\omega \cos \phi \cdot r+\left\{\omega^{2} \cos ^{2} \phi \cdot r^{3}-\cot \phi\left(\frac{1}{\rho} \frac{\partial p}{\partial \phi}\right)\right\}^{1 / 2}
$$

the negative sign before the radical being omitted by the above reasoning. It should be noted that $v_{\lambda}+\omega \cos \phi \cdot r>0$. Now the linear velocity due to the rotation of the earth at the latitude $\phi$ is $\omega \cos \phi \cdot r$. Thus we arrive at the result that the solution of the gradient wind equation which is significant represents a zonal motion whose absolute velocity is positive in space.

In dealing with the zonal motion, it is frequently advantageous to use the $a b-$ solute angular momentum $\Omega$ as follows:

$$
\Omega=r \cos \phi\left(v_{\lambda}+r \cos \phi \cdot \omega\right) . \quad(\tilde{5} \cdot a)
$$

When the west-east component of the pressure gradient $\partial p / \partial \lambda$ vanishes, the equation (1) degenerates into a simple equation
In dealing with the zonal motion, it is frequently advantageous to use the vertical component of the absolute vorticity or simply the absolute vorticity $Z$ as follows: $Z=\frac{\partial v_{\phi}}{r \cos \phi \partial \lambda}-\frac{\partial v_{\lambda}}{r \partial \phi}+\tan \phi \frac{v_{\lambda}}{r}+2 \sin \phi,(5 . \mathrm{b})$ or $Z=\xi+2 \omega \sin \phi$, where $\xi$ is the relative vorticity. For an incompressible atmosphere, upon eliminating the pressure from (1) and (2), we find 


$$
\frac{d \Omega}{d t}=0 . \quad \text { (6. a) } \quad \frac{d Z}{d t}=0 .
$$

Although the velocity $v_{\lambda}$ changes with the latitude for the moving mass of air, the angular momentum remains constant, if the zonal component of the pressure gradient is zero. To follow the motion of a particle of air, the angular-momentum is one of conservative properties, under certain conditions.
Although the relative vorticity changes with the latitude for the moving mass of air, the absolute vorticity remains constant, if the air is incompressible. To follow the motion of a particle of air, the absolute vorticity is one of conservative properties, under certain conditions.

For the zonal circulation of the atmosphere, the two conservative properties can be written as:

$$
\Omega=r \cos \phi\left(v_{\lambda}+r \cos \phi \cdot \omega\right) \text {. (7.a) } \quad, \quad Z=-\frac{\partial v_{\lambda}}{r \partial \phi}+\tan \phi \frac{v_{\lambda}}{r}+2 \omega \sin \phi .
$$

There is an important relation between these two quantities for the zonal current:

$$
Z=-\frac{1}{r^{2} \cos \phi} \partial \Omega
$$

If a mass is brought from a latitude $\phi_{0}$ to another latitude $\phi$ at the same level under conservation of its angular momentum, we have

$$
\cos \phi\left(v_{\lambda}+\omega r \cos \phi\right)=\cos \phi_{0}\left(v_{\lambda_{0}}+\omega r \cos \phi\right) .
$$

The linear velocity relative to the earth in the west-east direction at the latitude $\phi$ is, therefore, given by

$$
v_{\lambda}=\frac{1}{\cos \phi}\left\{v_{\lambda_{0}} \cdot \cos \phi_{0}+\omega r\left(\cos ^{2} \phi_{0}-\cos ^{2} \phi\right)\right\},
$$

or

$$
v_{\lambda}=\frac{1}{\cos \phi}\left\{v_{\lambda 0} \cdot \cos \phi_{0}+\omega r\left(\sin ^{2} \phi-\sin ^{2} \phi_{0}\right)\right\}
$$$$
\left(9^{\prime}\right. \text {. a) }
$$

where $v_{\lambda 0}$ is the linear velocity relative to the earth from the west at the latitude $\phi_{0}$. When a mass originally at rest $\left(v_{\lambda_{0}}=0\right)$ is moved horizontally from a given latitude
To find the zonal wind distribution having a given absolute vorticity $Z_{0}$, from Eq. (7.b), we must solve the following differential equation:

$$
-\frac{1}{r} \frac{\partial \psi_{\lambda}}{\partial \phi}+\tan \phi \frac{v^{2 \lambda}}{r}+2 \omega \sin \phi=Z_{0},
$$

or

$$
-\frac{1}{r^{2} \cos \phi} \frac{\partial}{\partial \phi}\left(r \cos \phi \cdot v_{\lambda}+r^{2} \cos ^{2} \phi \cdot \omega\right)=Z_{i} .
$$

The solution for this equation is given by

$$
\begin{array}{r}
v_{n}=\frac{1}{\cos \phi}\left\{\cos \phi_{0} \cdot v_{\lambda_{0}}+\left(\sin \phi_{0}-\sin \phi\right) Z_{0} y^{r}\right. \\
\left.+\left(\cos ^{2} \phi_{0}-\cos ^{2} \phi\right) r \omega\right\}, \quad(9 . b)
\end{array}
$$

or

$$
\begin{array}{r}
v_{\lambda}=\frac{1}{\cos \phi}\left\{\cos \phi_{0} \cdot v_{\lambda 0}+\left(\sin \phi_{0}-\sin \phi\right) Z_{0}{ }^{2}+\right. \\
\left.\left(\sin ^{2} \phi-\sin ^{2} \phi_{0}\right) r \omega\right\}, \quad\left(9^{\prime} \cdot b\right)
\end{array}
$$
an equalization of absolute vorticity and the resulting zonal wind distribution will be given by Eq. (9. b). It is assumed here that if a mass is brought from a latitude $\phi_{0}$ 
$\phi_{0}$, the resulting velocity at the latitude $\phi$ becomes

$v_{\lambda}=\omega r\left(\sin ^{2} \phi-\sin ^{2} \phi_{0}\right) / \cos \phi .(10 . a)$ to another latitude $\phi$ at the same level under conservation of its absolute vorticity, the resulting velocity at the latitude $\phi$ is given by $E_{q}$. (9.b). When a omass riginally at rest $\left(v_{\lambda 0}=0, Z_{0}=2 \omega \sin \phi_{0}\right)$ is moved horizontally from a given latitude $\phi_{0}$, the resulting velocity at the latitude $\phi$ becomes, in virtue of the above assumptions,

$$
v_{\lambda}=\omega r\left(\sin \phi-\sin \phi_{0}^{2} / \cos \phi .(10 . b)\right.
$$

The essential difference between the equations (10.a) and (10.b) is that the term in brackets is the difference of two positive quantities in the former equation, while in the latter the resulting velocity is always positive.

The following Table 1.a shows the velocities that result when a mass originally at rest $\left(v_{\lambda 0}=0\right)$ is moved horizontally from a given latitude towards the pole or equator under conservation of its angular momentum, as given by EXNER (1933) and HAURWITZ (1941). The positive sign means a velocity towards the east, and the negative sign a westward velocity. Such high velocities, especially at higher latitudes, are rarely, if ever, observed in the atmosphere. It must, therefore, be concluded that large meridional displacements of air masses under conservation of angular momentum hardly occur in the atmosphere, at least in higher lati tudes.

Table 1.a

\begin{tabular}{c|c|c}
$\begin{array}{c}\text { Original } \\
\text { latitude, } \\
\phi_{0} \text { in } \\
\text { degrees }\end{array}$ & $\begin{array}{c}\text { Displacement } \\
\text { of } \begin{array}{c}10^{\circ} \text { towards } \\
\text { the pole } \\
\text { m/sec }\end{array}\end{array}$ & $\begin{array}{c}\text { Displaceme } \\
\text { of } \\
\text { the equator } \\
\text { m/sec }\end{array}$ \\
\hline $0^{\circ}$ & +14 & $\ldots \ldots$ \\
$10^{\circ}$ & +43 & -14 \\
$20^{\circ}$ & +71 & -41 \\
$30^{\circ}$ & +99 & -66 \\
$40^{\circ}$ & +125 & -87 \\
$50^{\circ}$ & +151 & -105 \\
$60^{\circ}$ & +180 & -118 \\
$70^{\circ}$ & +232 & -123 \\
$80^{\circ}$ & $+\infty$ & -118 \\
$90^{\circ}$ & $\ldots .$. & -80 \\
\hline
\end{tabular}

The following Table $1 . b$ shows the velocities that result when a mass originally at rest $\left(v_{\lambda_{0}}=0, Z_{0}=2 \omega \sin \phi_{0}\right)$ is moved horizontally from a given latitude towards the pole or the equator under conservation of its absolute vorticity, in virtue of the above assumptions. In connection with the following table, it is of interest to note that quite reasonable westerly winds may result from a meridional displacement. It must, therefore, be concluded that large meridional displacements of air mass under conservation of total vorticity are more probable in the atmosphere. One exception is the case for the terminal latitude $\phi=90^{\circ}$

Table 1.b

\begin{tabular}{|c|c|c|}
\hline $\begin{array}{c}\text { Original } \\
\text { latitude, } \\
\phi_{0} \text { in } \\
\text { degrees }\end{array}$ & $\begin{array}{l}\text { Digplacement } \\
\text { of } 10^{\circ} \text { towards } \\
\text { the pole } \\
\text { in/sec }\end{array}$ & $\begin{array}{c}\text { Displacement } \\
\text { of } 10^{\circ} \text { toward } \\
\text { the equator } \\
\mathrm{m} / \mathrm{sec}\end{array}$ \\
\hline $0^{\circ}$ & +14 & ...... \\
\hline $10^{\circ}$ & +14 & +14 \\
\hline $20^{\circ}$ & +13 & +13 \\
\hline $30^{\circ}$ & +12 & +12 \\
\hline $40^{\circ}$ & +11 & +11 \\
\hline $50^{\circ}$ & +9 & + \\
\hline $60^{\circ}$ & +7 & + \\
\hline $70^{\circ}$ & +5 & + \\
\hline $80^{\circ}$ & $+\infty$ & +3 \\
\hline $90^{\circ}$ & & +0.6 \\
\hline
\end{tabular}


When a mass whose initial linear velocity $v_{\lambda_{0}}$ is $10 \mathrm{~m} / \mathrm{sec}$ is moved horizontally from a given latitude $\phi_{0}=30^{\circ}$ towards the north or south under conservation of its angular momentum, the resulting velocity $v_{\lambda}$ at the latitude $\phi$ becomes

$$
v_{\mathrm{A}}=\frac{1}{\cos \phi}\left\{8.66+\omega r\left(\sin ^{2} \phi-0.25\right)\right\} \dot{\mathrm{m}} / \mathrm{sec}
$$

Suppose the basic flow with no relative vorticity as $V_{\lambda}=8.66 / \cos \phi(\mathrm{m} / \mathrm{sec})$, because of $\partial\left(r \cos \phi \cdot V_{\lambda}\right) / \partial \phi=0$. The excess wind speed $v_{\lambda}^{\prime}=v_{\lambda}-V_{\lambda}$ that results in higher and lower latitudes becomes so high with the increase of displaced distance (as shown in the annexed table), that meridional displacement of air particle under conservation of its angular momentum would hardly occur in the atmosphere.

\begin{tabular}{cccc}
\hline Latitude & $v_{\lambda}(\mathrm{m} / \mathrm{sec})$ & $V_{\lambda}(\mathrm{m} / \mathrm{sec})$ & $v_{\lambda}{ }^{\prime}(\mathrm{m} / \mathrm{sec})$ \\
\hline $20^{\circ}-56.32$ & 9.22 & -65.54 \\
$25^{\circ}$ & -26.92 & 9.56 & -36.48 \\
$30^{\circ}+10.00$ & 10.00 & 0.00 \\
$35^{\circ}+55.22$ & 10.57 & 44.65 \\
$40^{\circ}+109.95$ & 11.31 & 98.64
\end{tabular}

When a mass, whose initial linear velocity is $10 \mathrm{~m} / \mathrm{sec}$ and initial absolute vorticity $2 \omega \sin \phi_{0}$, is moved horizontally from a given latitude $\phi_{0}=30^{\circ}$ towards the north or south under conservation of its absolute vorticity, the resulting velocity $v_{n}$ at the latitude $\phi$ becomes, in virtue of theabove assumptions,

$$
v \lambda=\frac{1}{\cos \phi}\left\{8.66+\omega r(\sin \phi-0.50)^{2}\right\} \mathrm{m} / \mathrm{sec} .
$$

Suppose the basic flow with no relative vorticity $V_{\lambda}=8.66 / \cos \phi(\mathrm{m} / \mathrm{sec})$. The excess wind speed $v_{\lambda}{ }^{\prime}=v_{\lambda}-V_{\lambda}$ that results in higher and lower latitudes is rather small. and is surprisingly symmetric with respect. to the original latitude as shown in the annexed table.

\begin{tabular}{cccc}
\hline Latitude & $v_{\lambda}(\mathrm{m} / \mathrm{sec})$ & $V_{\lambda}(\mathrm{m} / \mathrm{sec})$ & $v_{\lambda}{ }^{\prime}(\mathrm{m} / \mathrm{sec})$ \\
\hline $20^{\circ}$ & 21.52 & 9.22 & 12.30 \\
$25^{\circ}$ & 12.61 & 9.56 & 3.05 \\
$30^{\circ}$ & 10.00 & 10.00 & 0.00 \\
$35^{\circ}$ & 13.64 & 10.57 & 3.07 \\
$40^{\circ}$ & 23.64 & 11.31 & 12.33 \\
\hline
\end{tabular}

Next, the author would like to mention the zonal circulation with constant angular momentum given by FERREL and the zonal circulation with constant absolute vorticity given by ROSSBY for the special interests.

FERREI, derived his well-known expression for the zonal circulation

$$
v_{\lambda}=r \omega\left(\frac{2}{3}-\cos ^{2} \phi\right) / \cos \phi,
$$

under the assumption that the whole atmos. phere was originally at rest and then the distribution of zonal wind with respect to latitudes is controlled by a large-scale lateral mixing process conserving the angular momentum. Putting

$$
v_{\lambda 0}=0 \text { at } \phi_{0}=35^{\circ} 16^{\prime} \text {, or } \cos ^{3} \phi_{0}=\frac{2}{3} \text {, }
$$

in $\mathbf{E q} .(9 \mathfrak{a})$, we get the last expression.

Continued lateral mixing would result. in the establishment of a zonal wind profile. chracterized by a constant absolute vorticity. Putting $v_{\lambda 0}=$ finite, $Z_{0}=2 \omega$ at the polar air cap $\phi_{0}=90^{\circ}$ in Eq. (9. b), we get

$$
\begin{gathered}
v_{\lambda}=r \omega(1-\sin \phi)^{3} / \cos \phi, \text { or } \\
v_{\lambda}=r \omega \cos \phi \cdot(1-\sin \phi) /(1+\sin \phi) .
\end{gathered}
$$

The last expression was given by $C_{*}-G_{*}$ ROSSBY (1947) for the zonal circulation with constant vorticity under the influence of large scale horizontal mixing processes.

The zonal circulation of the atmosphere expressed by the last formula is the circu- 
The latitude $35^{\circ} 16^{\prime}$ corresponds to the high belt in middle latitudes. lation characterized by a constant potential vorticity.

\section{§ 3. Meridional displacements of air masses under conservation of angular momentum and absolute vorticity.}

Thus, it may be seen from the above tables that the angular-momentum-transfer method can hardly describe large meridional displacements of air masses in the atmosphere and that the vorticity-transfer method can supply better estimation. Next, small meridional displacements of air will be examined from both points of view.

The angular momentum of a mass is constant, if the fluctuating variations of pressure gradient in the west-east direction, which certainly exist in a turbulent field of How, are ineffective. If the air particle is displaced from a latitude $\phi-d \phi$ to another latitude $\phi$ while retaining its angular momentum, its velocity becomes $v_{\Lambda}{ }^{*}$ at the latitude $\phi$ which is given by $r \cos \phi\left\{v^{*}(\phi)+\omega r \cos \phi\right\}$

$=r \cos (\phi-d \phi)\{v(\phi-d \phi)+\omega r \cos (\phi-d \phi)\}$. Expanding $\cos (\phi-d \phi)$ and $v(\phi-d \phi)$ in a series of powers of the small quantity $d \phi$ with coefficients which are functions of latitude $\phi$ and neglecting small quantities of the second order, we get the excess wind speed of the displaced air $v_{\lambda}^{\prime}$ as

$$
\begin{aligned}
v_{\lambda}{ }^{\prime} & =v_{\lambda}^{*}(\phi)-v_{\lambda}(\phi) \\
& =\left(-\frac{\partial v_{\lambda}}{r \partial \phi}+\frac{v_{\lambda}}{r} \tan \phi+\Omega \omega \sin \phi\right) \cdot r d \phi .
\end{aligned}
$$

Csing formulae (7.b) and (8), the last equation becomes

$$
v_{\lambda}{ }^{\prime}=Z \cdot r d \phi,
$$

or

$$
v_{\lambda}^{\prime}=-\frac{\partial \Omega}{r^{2} \cos \phi \partial \phi} \cdot r d \phi .\left(11^{\prime} \cdot a^{\prime}\right)
$$

This value of the excess west wind of the
The absolute vorticity of a mass is constant, if the atmosphere is incompressible. If the particle is displaced from a latitude $\phi_{0}$ to another latitude $\phi$ while retaining its absolute vorticity, its linear velocity becomes $v^{*}$ at $\phi$, which is given by, in virtue of tho above assumptions [from Eq. (9.b)],

$$
\begin{aligned}
& v_{\lambda}^{*}(\phi)=\frac{1}{\cos \phi}\left\{\cos (\phi-d \phi) \cdot v_{\lambda}(\phi-d \phi)\right. \\
& +[\sin (\phi-d \phi)-\sin \phi] Z(\phi-d \phi) \cdot r \\
& \left.+\left[\cos ^{2}(\phi-d \phi)-\cos ^{2} \phi\right] r \omega\right\} .
\end{aligned}
$$

Expanding $\cos (\phi-d \phi), \sin (\phi-d \phi), v_{\wedge}(\phi-$ $d \phi)$ and $Z(\phi-d \phi)$ in a series of powers of the small quantity $d \phi$, and neglecting small quantities of the third order, we get the excess wind speed $v_{\lambda}^{\prime}$ as

$$
v_{\lambda}^{\prime}=v_{\lambda}{ }^{*}(\phi)-v_{\lambda}(\phi)={ }_{2}^{1} \frac{\partial Z}{r \partial \phi} \cdot(r d \phi)^{2},
$$

where

$$
\begin{array}{r}
\frac{\partial Z}{\partial \phi \phi}=\frac{1}{r^{2}}-\frac{\partial^{2} v_{\lambda}}{\partial \phi^{2}}++\tan \phi \cdot \frac{\partial v_{\lambda}}{\partial \phi} \\
\left.+\frac{v_{\lambda}}{\cos ^{2} \phi}+2 \omega r \cos \phi\right\} .
\end{array}
$$

This value of the excess west wind of the displaced air is at least an approximation 
displaced air is at least an approximation to the turbulent velocity component. As the angular momentum usually decreases with increasing latitude in the (dynamic stable) atmosphere, so the displaced particle moving towards the pole gains the west component of wind speed, and the particle moving towards the equator Ioses the west component of wind speed (see Table 1.a). The excess wind speed under conservation of its angular momentum, is a small quantity of the first order with respect to the displaced distance $(r d \phi)$. to the turbulent velocity. As the absolute vorticity is nearly equal to $2 \omega \sin \phi$ in the normal atmosphere, the displaced particle moving north or south gains the west component of wind speed (see Table 1.b). The excess west wind speed under conservation of its absolute vorticity is the small quantity of the second order with respect to the displaced distance $(r d \phi)$.

The potential vorticity $\xi_{0}$ after ROSSBY (1940) represents the relative vorticity that the air particle would have if it were brought under conservation of its absolute vorticity to a standard latitude $\phi_{0}$, or $\xi_{0}=\xi+2 \omega\left(\sin \phi-\sin \phi_{0}\right)$. When the potential vorticity increases, decreases or is constant with latitude,

i. e., $\quad \partial Z / \partial \phi\rangle,\langle$, or $=0$, the excess wind speed of the displaced particle under conservation of its absolute vorticity will be positive (toward the east), negative. (toward the west) or zero.

\section{References}

(1) EXNER, FELIX M., 1925: Dynamische Meteorologie, zweite Aufl., Wien, 22-25 und 249-252.

(2) HAURWITZ, B,, 1941: Dynamic Meteorology, MoGrav-Hill Book Company, 119-121 and $292-295$.

(3) ROSSBY, C.-G., 1940: Planetary Flow Patterns in the Atmosphere, Quarterly Journal of Roy. Met. Soc, 66, Supplement, 68-87. under the Intluence of Large-scale Horizontal Mixing Processes, Bull. Amer. Met. Soc, 28, $53-68$. 